\title{
Inferomedially impacted zygomatic fracture reduction by reverse vector using an intraoral approach with Kirschner wire
}

\author{
Jin Woo Jang ${ }^{1}$, Jaeyoung Cho ${ }^{1}$, Jin Sik Burm ${ }^{2}$ \\ ${ }^{1}$ Department of Plastic Surgery, Kyung Hee University Hospital, Seoul; ${ }^{2}$ Department of Plastic Surgery, Kyung Hee University Hospital, \\ Kyung Hee University College of Medicine, Seoul, Korea
}

\begin{abstract}
Background In inferomedially rotated zygomatic fractures sticking in the maxillary sinus, it is often difficult to achieve complete reduction only by conventional intraoral reduction. We present a new intraoral reduction technique using a Kirschner wire and its clinical outcome. Methods Among 39 inferomedially impacted zygomatic fractures incompletely reduced by a simple intraoral reduction trial with a bone elevator, a Kirschner wire $(1.5 \mathrm{~mm})$ was vertically inserted from the zygomatic body to the lateral orbital rim in 17 inferior-dominant rotation fractures and horizontally inserted to the zygomatic arch in nine medial-dominant and 13 bidirectional rotation fractures. A Kirschner wire was held with a wire holder and lifted in the superolateral or anterolateral direction for reduction. Following reduction of the zygomaticomaxillary fracture, internal fixation was performed.

Results Fractures were completely reduced using only an intraoral approach with Kirschner wire reduction in 33 cases and through an additional lower lid or transconjunctival incision in six cases. There were no surgical complications except in one patient with undercorrection. Postoperative 6-month computed tomography scans showed complete bone union and excellent bone alignment. Four patients experienced difficulty with upper lip elevation; however, these problems spontaneously resolved after manual tissue lump massage and intralesional steroid (Triamcinolone) injection.

Conclusions We completely reduced infraorbital rim fractures, zygomaticomaxillary buttresses, and zygomaticofrontal suture fractures in $84 \%$ of patients through an intraoral approach alone. Intraoral Kirschner wire reduction may be a useful option by which to obtain effective and powerful reduction motion of an inferomedially rotated zygomatic body.
\end{abstract}

Keywords Zygomatic fracture / Rotation / Zygoma
Correspondence: Jin Sik Burm Department of Plastic Surgery, Kyung Hee University Hospital, Kyung Hee University College of Medicine, 23 Kyungheedae-ro, Dongdaemun-gu, Seoul 02447, Korea

Tel: +82-2-958-8431

Fax: +82-2-963-5638

E-mail: jsburm@gmail.com

Received: November 10, 2020 • Revised: December 21, 2020 • Accepted: December 29, 2020

pISSN: 2234-6163 • elSSN: 2234-6171 • https://doi.org/10.5999/aps.2020.02173• Arch Plast Surg 2021;48:69-74

\section{INTRODUCTION}

The inferomedially rotated zygomatic fracture is a common type of zygomatic fracture [1-3], in which the inferomedial frac- ture margin of the zygomatic body is almost always stuck in the maxillary sinus with comminuted fracture segments of the maxilla, especially at the zygomaticomaxillary buttress. Thus, it is also called the inferomedially impacted zygomatic fracture. To

Copyright $(\odot) 2021$ The Korean Society of Plastic and Reconstructive Surgeons

This is an Open Access article distributed under the terms of the Creative Commons Attribution Non-Commercial License (https://creativecommons.org/

licenses/by-nc/4.0/) which permits unrestricted non-commercial use, distribution, and reproduction in any medium, provided the original work is properly cited.

www.e-aps.org 
achieve the strongest reduction motion of the fractured zygoma according to the lever law, the ideal application point of a reduction force is the inferior or medial portion of the zygomatic body. This point is furthest from the hinge, which is the zygomaticofrontal suture or zygomatic arch.

In conventional techniques adopting the intraoral approach with upper gingivobuccal sulcus incision, a bone elevator [3-6] or bone hook [7] is inserted underneath the zygomatic body, and anterolateral traction force is applied. The application point of the bone elevator is commonly the posterior surface of the zygomatic body in the most anterior portion of the zygomatic arch, which is close to the hinges. The traction force at this point must be stronger than the force required to reduce the impacted zygomaticomaxillary fracture furthest from the hinges. Therefore, if the inferomedial portion of the fractured zygoma body is severely stuck in the maxillary sinus, it is often difficult to achieve complete anatomical reduction by only conventional bone-elevator reduction, especially in medial rotation fractures, resulting in need for an additional powerful and effective reduction force. In zygomatic reduction with a bone hook, the hooking point is located on the medial or inferior fracture margin of the zygomatic body farthest from a hinge or at the zygomatic recess of the maxillary sinus. Therefore, the theoretical required reduction force is equal to the actual force for complete reduction motion of the zygoma, resulting in a more accurate and effective reduction with a lower force than seen in bone elevator reduction. However, the strong hook insertion or traction procedure often causes additional bone fracture or displacement or fragmentation of the comminuted zygomaticomaxillary fracture and can cause difficult bone fixation as well as additional sinus injury and its related complications.

To achieve the effective and powerful force necessary for complete reduction and fixation of the zygoma as well as to avoid additional soft tissue or bone injury, we designed a new surgical procedure in which a thick Kirschner $(\mathrm{K})$ wire is inserted into the medial or inferior portion of the zygomatic body furthest from a hinge and raised in the anterolateral or superolateral direction. The goal of this study was to present an intraoral K-wire reduction technique for use in inferomedially rotated zygomatic fractures in the same operative field and to evaluate the clinical results.

\section{METHODS}

\section{Patients}

Inferomedially rotated zygomatic fractures were in 63 of the 82 patients with zygomatic fracture who underwent surgery from January 2015 to December 2019. Among them, intraoral K-wire reduction was performed in 39 inferomedially impacted fractures, in which the zygomatic body was incompletely reduced by a simple reduction technique with a bone elevator through an intraoral approach. These zygomatic fractures were classified as follows according to the dominant rotational direction of the zygomatic body on preoperative facial three-dimensional computed tomography (3D CT) images to determine the direction of K-wire insertion in advance: 17 fractures with inferior-dominant rotation, nine fractures with medial-dominant rotation, and 13 fractures with inferior and medial bidirectional rotation. All fractures had mild fracture displacement or green stick fracture of the zygomatic arch or zygomaticofrontal suture with or without mild comminution. However, severe depressed quadripod fracture in inferior-dominant rotation type with severe fracture displacement of zygomatic arch or zygomaticofrontal suture were excluded from this study. Preoperative, immediately postoperative, and 6-month follow-up facial 3D CT images were collected for evaluation of case outcomes. An independent physician evaluated the degree of zygomatic bone symmetry based on the 3D CT images. The patients also were asked the degree of malar symmetry using the same scale by seeing their zygoma through a mirror at the last follow-up. The scale was excellent for no asymmetry, good for minimal asymmetry or depression, poor for moderate asymmetry or depression.

The study was approved by the Institutional Review Board of Kyung Hee University Hospital (IRB No. 2020-10-040) and performed in accordance with the principles of the Declaration of Helsinki. The patients provided written informed consent for the publication and the use of their images.

\section{Surgical technique}

The zygomaticomaxillary fracture was fully exposed through an intraoral approach with an upper gingivobuccal sulcus incision. First, the bone elevator was inserted underneath the zygomatic body in the most anterior portion of the zygomatic arch. Next, superolateral or anterolateral traction force was applied for initial bone reduction. We checked for incomplete reduction status of the comminuted zygomaticomaxillary fracture on the infraorbital rim, anterior maxillary wall, and zygomaticomaxillary buttress related to the dominant rotation direction of the zygomatic body.

A thick K-wire (1.5 $\mathrm{mm}$ in diameter) was inserted into the thick zygomatic body near the fracture line, furthest from the hinge of the rotation fracture, to about $4 \mathrm{~cm}$ deep, with a drilling device applied in the same operative field of the intraoral approach (Fig. 1). In inferior-dominant rotation fractures, the wire was inserted vertically from the inferior area of the zygomatic body to the lateral orbital rim. In medial-dominant or bidirec- 


\section{Fig. 1. Insertion direction of intraoral K-wire}

A thick Kirschner wire (K-wire) was inserted at the thick zygomatic body near the fracture line furthest from the rotation hinge. In inferior-dominant rotation fracture, (A) vertical K-wire insertion from the zygomatic body to the lateral orbital rim and (B) lifting up for superolateral reduction. In medial-dominant or bidirectional rotation fractures, (C) horizontal insertion from the zygomatic body to the zygomatic arch and (D) lifting up for anterolateral reduction.
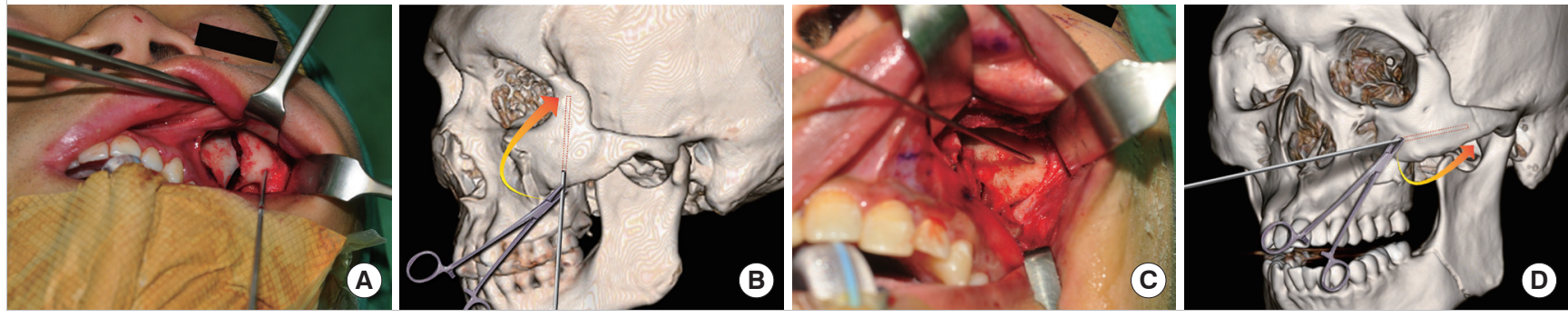

tional rotation fractures, it was horizontally inserted from the medial area of the zygomatic body to the zygomatic arch. This insertion point and direction were chosen to ensure that reduction rotation force was maximally powerful and effective in the direction opposite the rotation vector of the zygomatic fracture. For zygoma reduction, the K-wire was fixed near the insertion point with a wire holder and then was lifted superolaterally in inferior-dominant rotation fractures and anterolaterally in medial-dominant or bidirectional rotation fractures, while an assistant stabilized the head (Fig. 1).

After the zygomatic body was fully reduced, the fracture segments of the maxilla were gently and completely reduced from the maxillary sinus with a single skin hook, while slight over-reduction of the zygomatic body was maintained by lifting the $\mathrm{K}$ wire superolaterally or anterolaterally. Reduction alignment of the other fracture-displacement sites of the infraorbital rim, the zygomatic arch, and the zygomaticofrontal suture was confirmed by manual palpation or periosteal elevator manipulation. Subsequently, the zygomaticomaxillary buttress and the anterior maxilla were fixed with titanium microplates (1.2 mm Leibinger upper-face fixation module; Stryker, Kalamazoo, MI, USA) or absorbable polymer plates (1.5 mm CPS; Inion, Tampere, Finland), while complete reduction was maintained by holding a Kwire. When the infraorbital rim fracture was not reduced sufficiently, it was reduced and fixed with a plate or a wire through a transconjunctival or subciliary incision. Finally, the K-wire was removed by pulling it out manually or with a wire holder. The gingivobuccal incision was closed with 5-0 absorbable sutures. The compressive gauze dressing was applied along the nasolabial fold for three days after surgery to avoid development of dead space between the reduced bone and the elevated periosteum.

\section{RESULTS}

A total of 39 patients ( 35 men and four women: mean age, 35.5
Table 1. Patients demographics

\begin{tabular}{lc}
\hline Variable & Value $(\mathrm{n}=39)$ \\
\hline Age (yr) & $35.5(17-86)$ \\
Sex & 35 \\
Male & 4 \\
Female & \\
Cause & 18 \\
Slip down & 8 \\
Traffic accident & 6 \\
Assault & 7 \\
Others & \\
Combined fracture & 5 \\
Nasal bone & 34 \\
Orbital wall & 4 \\
Mandible & 3 \\
Le Fort I, II & 1 \\
Temporal bone & 1 \\
NEO & \\
Dominant rotation & 17 \\
Inferior & 9 \\
Medial & 13 \\
Bidirectional & \\
Fixation point & $33(84.61)$ \\
1 Point (B) & $6(15.38)$ \\
2 Points (B, R) & \\
Residual complication & 4 \\
Subcutaneous scar & 5 \\
Polypoid lesion/mucosal thickening & \\
\hline
\end{tabular}

Values are presented mean (range), number, or number (\%).

NEO, nasoethmoid-orbital; B, zygomaticomaxillary buttress; $R$, infraorbital rim.

years; age range, 17-86 years) with zygomaticomaxillary fracture was treated with open reduction and internal fixation. Fracture site (12 right and 27 left) and cause of injury (traffic accidents [driver, passenger, pedestrian; $n=8]$, assault $[n=6]$, fall $[\mathrm{n}=18$ ], trauma (explosion, sports activity, steel impact; $\mathrm{n}=7$ ]) were evaluated. Associated facial bone fractures included nasal bone fracture, orbital blowout fracture, Le Fort fracture (types 1 and 2), mandibular fracture, temporal bone fracture, and nasoethmoid-orbital fracture (Table 1). The follow-up period ranged 


\section{Fig. 2. Cases of intraoral K-wire reduction}

Preoperative and postoperative 6-month computed tomography (CT) images of inferior-dominant rotation fracture $(A, B)$, bidirectional rotation fracture $(C, D)$, and medial-dominant rotation fracture $(E, F)$. These zygomatic fractures were reduced through intraoral approach alone. Postoperative CT images show excellent bony alignment. K-wire, Kirschner wire.
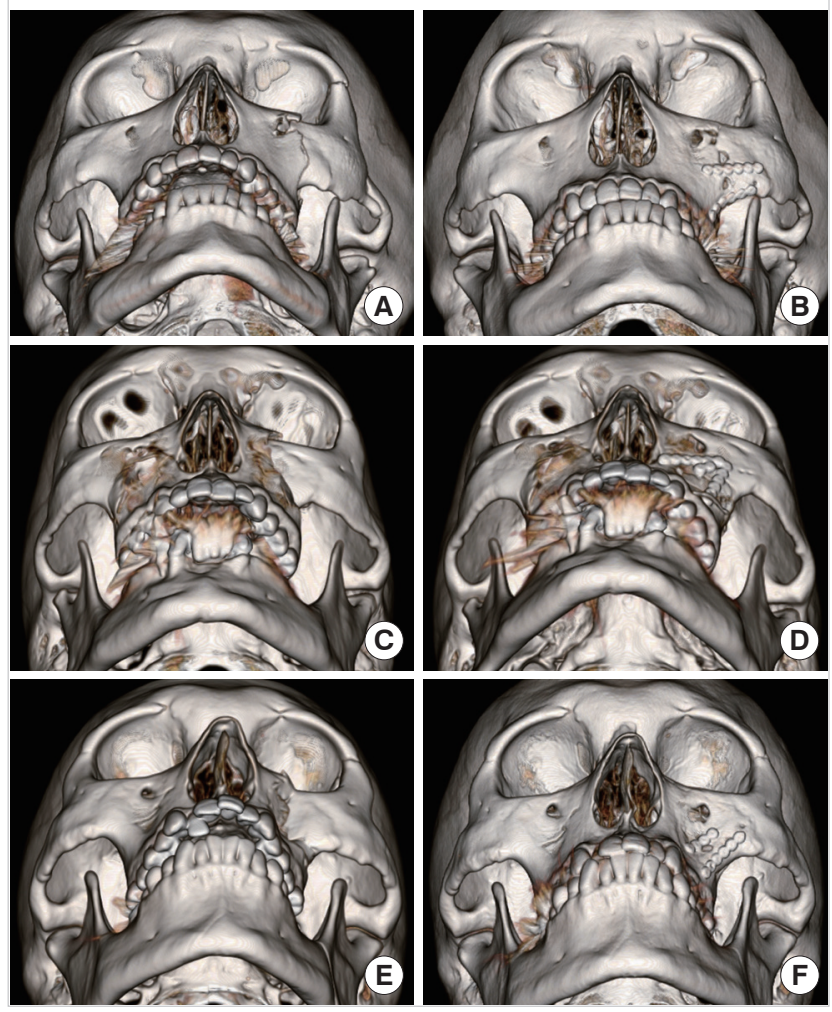

from 4 to 12 months (mean, 6.2 months).

Of the inferomedially impacted zygomatic fractures, 33 achieved complete reduction of the infraorbital rim fracture through an intraoral approach with K-wire reduction (84\%) and six through a lower lid or transconjunctival incision. Meanwhile, the zygomaticofrontal suture fracture was completely reduced through an intraoral approach with K-wire reduction in all cases. The number of fixation points on the quadripod was one (the zygomatic buttress) in 33 cases (84\%) and two (the zygomatic buttresses and the infraorbital rim) in six cases (16\%). The intraorbital rim fixation was done when segmental dislocation, fracture gap or unstable stepping of the infraorbital rim remained after intraoral reduction.

The operation time from incision to closure ranged from 45 to 121 minutes (mean, 68 minutes). The mean discharge time was 3.4 days postoperative. No early surgical complications were recorded. Immediate postoperative 3D CT imaging showed excellent reduction and symmetry of the zygomatic fractures, except in one case with comminution of the zygomatic body in which the malar prominence was mildly depressed due to un- dercorrection of its fracture segment.

During the follow-up period, 38 of 39 patients showed complete bone union and excellent bone alignment with no zygomatic asymmetry on a 3D CT scan (Fig. 2). Minimal depression of zygomatic body prominence was confirmed in one patient with comminuted zygomatic body fracture, but this patient evaluated malar symmetry as good appearance and did not request additional treatment.

Four patients complained of discomfort with subcutaneous scar tissue at the intraoral operation site after 2 to 3 weeks of operation, which resulted in limited upper lip elevation during smiling and consequential asymmetry. However, this issue spontaneously resolved after manual massage of scar tissue and one or two intralesional steroid (triamcinolone) injections. Mild polypoid lesion or mucosal thickening at the fracture site of the maxillary sinus was confirmed at final follow-up CT imaging in five patients, but all these cases were asymptomatic and involved no further reported issues.

\section{DISCUSSION}

The zygomatic bone has four legs, including the lateral orbital rim, the inferior orbital rim, the zygomaticomaxillary buttress, and the zygomatic arch. The most common fracture sites of the zygoma are the zygomaticomaxillary buttress and the inferior orbital rim, which are the weakest areas due to their bony thinness $[1,8,9]$. Therefore, the most common fracture type is believed to be the inferomedially rotated zygomatic fracture, which involves the lateral orbital rim and/or the zygomatic arch as the hinge [1-3]. In this fracture type, the inferomedial fracture margin of the zygomatic body is displaced and stuck in the maxillary sinus with the comminuted fracture segments of the anterior or lateral wall of the maxilla.

In our experiences conducting zygoma reduction through an intraoral approach, when the zygoma is severely stuck in the maxillary sinus or fracture displacement of the zygomaticofrontal suture or the zygomatic arch was observed, complete reduction of the zygoma often failed with a conventional reduction technique involving application of anterior and lateral traction forces with a bone elevator placed underneath the zygomatic body. This application point is located at the most anterior portion of the zygomatic arch posterolateral to the zygomatic body, rather than underneath the zygomatic body itself, close to the hinge, which is the zygomaticofrontal suture or the zygomatic arch. According to the law of the lever, the operator's traction force (effort) at this point close to the hinge must be stronger than the resistance (load) at the inferior or medial fracture site of the zygomatic body furthest from the hinge (fulcrum). 
Therefore, we thought that the main cause of reduction failure may be that the application point of the effect is too close to the hinge. If the application point were to be placed at the resistance point (fracture site), more effective and powerful reduction of the zygoma could be achieved with the same traction force applied at the conventional application point.

To apply a powerful traction force directly to the zygoma body in the same operative field via an intraoral approach, a few reduction techniques incorporating a bone hook or an intermaxillary fixation screw have been designed. Tarabichi [7] described a transsinus reduction technique with a bone hook inserted into the zygomatic recess of the maxillary sinus through a comminuted inferior fracture line. In our experiences, even though the bone hook reduction was powerful, it often produced additional fracture on the inferomedial fracture line of the zygomatic body and the comminuted bone segments of the maxilla during insertion and traction procedures. Moon et al. [10] described a minimally exposed zygomatic reduction technique with an intermaxillary fixation screw through an intraoral and subciliary approach with or without a lateral brow incision. They inserted one or two dual-top screws at the zygomatic body through an intraoral approach and, at the infraorbital rim through a subciliary approach, hung the wire on the screw head and pulled up a wire in the opposite direction of fracture displacement. This technique had a limitation in that it cannot completely reduce most fractures through an intraoral approach alone. In our experience, when the wire was pulled up, it often fell off the screw head.

The inferomedially rotated zygomatic fracture may be most effectively reduced when the reduction vector is opposite the direction of rotation displacement and its application point is furthest from the hinge. With our intraoral K-wire reduction technique, a K-wire is inserted from the medial portion of the zygomatic body to the zygomatic arch in medial-dominant rotation and medial and inferior rotation and from the inferior portion of the rigid zygomatic body to the lateral orbital rim in inferiordominant rotation. That is, in this context, a K-wire passes through the thickest part of the zygomatic body toward the hinge (rotation axis) of the rotated zygomatic fracture, and its longitudinal axis corresponds roughly to the rotation radius, which is the linear distance from its rotation axis to an application point of reduction force. Consequently, the application point of the K-wire traction vector for reduction is placed at the furthest distance from the hinge, and its direction is almost always perpendicular to the axis of the rotation radius. In addition, a K-wire penetrates two cortex of the anterior and posterolateral walls of the zygoma and passes just lateral to the lateral orbital rim in vertical insertion and just below the zygomatic arch in horizontal insertion. In this K-wire insertion area, there is no important structure such as the frontal branches of facial nerve or orbital contents. The K-wire did not disturb direct visibility of the operation field because of its thickness and insertion direction. Furthermore, there was no case of a problem because of overlapping between a K-wire insertion point and the plate fixation points. In vertical K-wire insertion for inferior-dominant rotation fracture, its insertion point was placed inferomedial to the zygomatic prominence, which rarely overlapped with the plate fixation points. In these cases, a plate was placed just beside a thin K-wire and fixed with no problem. In horizontal insertion for medial or bidirectional rotation fractures, its insertion point was placed medial to the zygomatic body, which did not overlap with any plate fixation points. Therefore, this technique allows reduction force to be applied powerfully, effectively, and safely in the opposite direction to rotation displacement of the zygoma without additional damage to the fracture site and strongly pulling out of a K-wire during the reduction procedure.

An infraorbital rim fracture can be easily reduced during anterolateral traction of a horizontally inserted K-wire with a wire holder. In addition, K-wire traction can cause the comminuted depressed segments of the maxilla to be completely reduced from the maxillary sinus and effectively fixed to the zygomatic body and the intact maxilla by maintaining the reduction state of the zygomatic body. In this study, even though the inferomedially rotated zygomatic fracture was incompletely reduced by an initial reduction trial with a bone elevator, we were able to completely reduce the infraorbital rim fracture in $84.7 \%$ of cases and the zygomaticomaxillary buttress and the zygomaticofrontal suture fracture in 100\% of cases through an intraoral approach alone. In addition, this technique can be applied to reduction of inferolaterally rotated zygomatic fractures by vertical K-wire insertion and superomedial traction.

The disadvantage inherent with intraoral K-wire reduction is wide exposure of the zygomatic body and the anterior maxilla for inserting a K-wire into the zygomatic body and for reducing the infraorbital rim fracture as well as the zygomaticomaxillary buttress fracture. This wide periosteal elevation can induce subperiosteal dead space; periosteal detachment; and consequent loosening of levator muscles of the upper lip, including the zygomaticus muscles, resulting in drooping of the cheek or upper lip, a shallow nasolabial fold, or unnatural smile [11]. To prevent the onset of this problem, we applied a compressive gauze dressing to the nasolabial fold for three days after surgery to avoid development of dead space between the reduced bone and the elevated periosteum, especially on the concave zygomaticomaxillary buttress anteroinferior to the prominent zygomatic body. In this study, subcutaneous scar tissue lumping occurred in four patients despite nasolabial compressive dressing, resulting in increased dif- 
ficulty with upper lip elevation and mild asymmetry. Fortunately, this discomfort was spontaneously resolved after tissue lump massage and one or two intralesional triamcinolone injections.

In conclusion, we applied a new technique for intraoral K-wire reduction to 39 inferomedially impacted zygomatic fractures, in which the zygomatic body was incompletely reduced by a simple reduction technique with a bone elevator using an intraoral approach. A K-wire was inserted from the zygomatic body to the hinge (rotation axis) vertically in cases of inferior-dominant rotation fracture or horizontally in cases of medial-dominant or bidirectional rotation fracture and then lifted in a superolateral or anterolateral direction. This technique allows reduction force to be applied powerfully, effectively, and stably in the opposite direction to rotation displacement of the zygoma without additional damage to the fracture site. We were able to completely reduce the infraorbital rim fracture, zygomaticomaxillary buttress, and the zygomaticofrontal suture fracture in $84 \%$ of cases through an intraoral approach alone. Six-month follow-up CT scans showed complete bone union and excellent bone alignment in 38 patients. Therefore, intraoral K-wire reduction may be a new option for powerfully, effectively, and stably reducing severe inferomedially rotated zygomatic fractures.

\section{NOTES}

\section{Conflict of interest}

No potential conflict of interest relevant to this article was reported.

\section{Ethical approval}

The study was approved by the Institutional Review Board of Kyung Hee University Hospital (IRB No. 2020-10-040) and performed in accordance with the principles of the Declaration of Helsinki. Written informed consent was obtained.

\section{Patient consent}

The patients provided written informed consent for the publication and the use of their images.

\section{Author contribution}

Conceptualization: JS Burm. Data curation, formal analysis: JW Jang. Methodology: JW Jang, J Cho. Project administration: JS Burm. Writing - original draft: JW Jang. Writing - review \& edit- ing: JS Burm.

\section{ORCID}

Jin Woo Jang

Jaeyoung Cho

https://orcid.org/0000-0002-5073-8666

https://orcid.org/0000-0003-1451-1824

Jin Sik Burm

\section{REFERENCES}

1. Hwang K, Kim DH. Analysis of zygomatic fractures. J Craniofac Surg 2011;22:1416-21.

2. Toriumi M, Nagasao T, Itamiya T, et al. 3-D analysis of dislocation in zygoma fractures. J Craniomaxillofac Surg 2014; 42:397-402.

3. Ji SY, Kim SS, Kim MH, et al. Surgical methods of zygomaticomaxillary complex fracture. Arch Craniofac Surg 2016;17: 206-10.

4. Birgfeld CB, Mundinger GS, Gruss JS. Evidence-based medicine: evaluation and treatment of zygoma fractures. Plast Reconstr Surg 2017;139:168e-180e.

5. Hollier LH, Thornton J, Pazmino P, et al. The management of orbitozygomatic fractures. Plast Reconstr Surg 2003;111: 2386-92.

6. Courtney DJ. Upper buccal sulcus approach to management of fractures of the zygomatic complex: a retrospective study of 50 cases. Br J Oral Maxillofac Surg 1999;37:464-6.

7. Tarabichi M. Transsinus reduction and one-point fixation of malar fractures. Arch Otolaryngol Head Neck Surg 1994; 120:620-5.

8. Kelley P, Hopper R, Gruss J. Evaluation and treatment of zygomatic fractures. Plast Reconstr Surg 2007;120(7 Suppl 2): 5S-15S.

9. Evans BGA, Evans GRD. MOC-PSSM CME article: zygomatic fractures. Plast Reconstr Surg 2008;121(1 Suppl): 1-11.

10. Moon SH, Lee JH, Oh DY, et al. Reduction of zygomatic fracture segment with intermaxillary fixation screw. J Craniofac Surg 2012;23:842-4.

11. Park BY, Song SY, Yun IS, et al. First percutaneous reduction and next external suspension with Steinmann pin and Kirschner wire of isolated zygomatic fractures. J Craniofac Surg 2010;21:1060-5. 